\title{
Neighborhood Social Environment and Health Communication at Prepregnancy and Maternal Stages among Caucasian and Asian Women: Findings from the Los Angeles Mommy and Baby Survey
}

\author{
Lu Shi ${ }^{1}$ and Yuping Mao ${ }^{2}$ \\ ${ }^{1}$ Department of Public Health Sciences, Clemson University, Clemson, SC 29634-0745, USA \\ ${ }^{2}$ Department of Media \& Communication, Erasmus University Rotterdam, 3000 DR, Netherlands \\ Correspondence should be addressed to Lu Shi; lushi.pku@gmail.com
}

Received 22 August 2014; Revised 16 November 2014; Accepted 3 December 2014; Published 29 December 2014

Academic Editor: Apostolos Vantarakis

Copyright (C) 2014 L. Shi and Y. Mao. This is an open access article distributed under the Creative Commons Attribution License, which permits unrestricted use, distribution, and reproduction in any medium, provided the original work is properly cited.

Introduction. We study whether the relationship between neighborhood social environment and maternal communication with healthcare providers differs between Asians and Caucasians. Method and Materials. Using the 2007 Los Angeles Mommy and Baby (LAMB) survey, we measure new mother's neighborhood social environment by four key variables: (1) instrumental/emotional support during pregnancy, (2) neighborhood social cohesion, (3) neighborhood social exchange, and (4) neighborhood services. Logistic regressions were applied for data analysis. Neighborhood social exchange predicts less chance of lacking communication about sensitive issues in preconception visits among Caucasians (logged odds: $-0.045 ; P<0.01$ ) and Asians (L.O.: -0.081 ; $P<0.001)$ and predicts less chance of lacking communication during preconception visits among Asians (L.O.: $-0.092 ; P<0.05)$. Neighborhood social cohesion predicts more chance for lacking communication about preparation for pregnancy only among Asians (L.O.: 0.065; $P<0.05$ ). Neighborhood services predict less chance of lacking communication about stigmatized issues in the prenatal visit among Asians (L.O.: $-0.036 ; P<0.05$ ). Discussion. Caucasians and Asians with more neighborhood social exchange are more likely to discuss sensitive issues during preconception visits. Neighborhood service significantly predicts maternal discussion of stigmatized issues with health care providers, but only among Asians.

\section{Introduction}

In developed nations, racial/ethnic disparities persist in receipt of a wide range of health services such as specialty care, pain assessment and treatment, and mental health services [1]. Asian women above 35 years are at higher risk of giving birth to a child with Down syndrome in comparison with Caucasian women in the same age group [2]. This risk could be effectively reduced by seeing an obstetrician prior to the pregnancy and obtaining recommended nutritional supplements such as folic acid, as well as by taking a prenatal screening to monitor the fetal growth. However, a study from the United Kingdom shows that South Asian women with positive attitudes toward taking prenatal screening for Down syndrome are less likely to take the test than
Caucasian women with positive attitudes toward the test [3]. The racial difference in receiving maternal health services is an important topic in racial/ethnic health disparities, since maternal health affects both women and their children's health. It is also a health service the majority of women will receive at least once in their lives. Thus, the disparity of receiving maternal health services between Asian pregnant women and non-Hispanic Caucasian (to be referred to as "Caucasian" hereinafter) pregnant women requires more research attention, given the severity of this disparity.

Some of the racial disparities between Asian women and Caucasian women in receiving healthcare services may be attributed to challenges associated with the former's intercultural communication with their healthcare providers, the economic difficulties during the immigration process, 
and the psychological challenges during the acculturation process. Female immigrants encounter various challenges such as economic strain, acculturation stress, and language barriers, which may negatively affect health [4]. As an additional challenge, sometimes the acculturation process seems to have a negative rather than positive health impact on maternal health, as is the case for many Southeast Asian immigrant women in Canada [5]. Some evidence from the United States even indicates that the general health status of certain Asian and Pacific Islander immigrants could deteriorate when their length of residency in the US increases [6]. These findings contradict the stereotype of "the model minority" and suggest that challenges to Asian immigrants' health might be underestimated and understudied.

Communication between healthcare providers and recipients is a potential contributing factor of disparities in health outcomes [7]. During women's prepregnancy and prenatal visits with healthcare providers, it is especially challenging for women and their healthcare providers to discuss socially stigmatized health topics that are important to the health of both mother and baby. Many barriers exist for Asian and Latino women in the US who seek help from healthcare providers when experiencing intimate partner abuse including social isolation, language barriers, discrimination and fears of deportation, dedication to the children and family unity, shame related to the abuse, and the cultural stigma of divorce [8]. HIV/AIDS positive status disclosure is one sensitive issue investigated in this study. One of few research papers on Asian population's HIV/AIDS disclosure found the communication barriers among the Asian population on this topic include fear of stigma, concerns of disappointing, burdening others, fear of discrimination, protection of family from shame, protection of family from obligation to help, and avoidance of communication regarding highly personal information $[9,10]$. Thus it is worthwhile to explore what might facilitate or hinder Asian women's communication about these sensitive issues with health care providers in the host country.

One possible factor that may have a dubious impact on women's communication with health care providers is the kind of social support they receive. Social support is a complex process involving "transactions between individuals and their social networks, for example, providing and receiving tangible (material) or intangible (emotional, informational, or cognitive guidance) support, and developing and maintaining support network resources" [11]. A lot of research has focused on the relationship between social support and particular medical maternal outcomes such as birth weight [12] and fetal growth [13]. It is also evident that, among women experiencing intimate partner violence, the more social support these women receive, the lower their risk of perceived poor mental health, physical health, anxiety, current depression, and suicide attempts [13]. During their interactions with healthcare providers, prospective mothers choose to share or not to share some important health information that might be considered as sensitive or private in their own cultures. This decision on communication with their healthcare providers and their health decision-making might be influenced by information and support that they receive from different sources such as neighbors and friends. For immigrant women who stay among fellow immigrants from one sending country, folk belief and folk medicine could displace health care utilization such as physician visits and thus serve as a roadblock to health outcome maximization [14]. As low social support has been shown to be associated with more health care utilization [15], it is reasonable to suspect that heightened social support for a mother could lower the likelihood of her prepregnancy and prenatal visits.

This study examines the relationship between social support and women's health communication with healthcare providers such as whether women make prepregnancy and maternal visits with their healthcare providers and to what extent women communicate sensitive issues (e.g., abuse and illegal drug use) as well as mental health issues (e.g., anxiety and depression) with their healthcare providers. In particular, our study examines the association of neighborhood social support with prospective mothers' health communication since neighborhood is an important and convenient source of social support for many people. Neighborhood characters and social support are related to various health issues. For instance, neighborhood cohesion has been found to positively correlate to youth participation in physical activity [16]. Buka et al. [17] reported that neighborhood-level factors are significantly associated with infant birth weight. In the existing literature on maternal and child health in relation to neighborhood context, the following categories of neighborhood characteristics have been addressed: income/wealth, employment, family structure, population composition, housing, mobility, education, occupation, social resources, violence and crime, deviant behavior, and physical conditions [18]. Only in more recent years, research has been conducted on neighborhood social support in relation to maternal health. Nkansah-Amankra et al's [19] research shows low neighborhood social support is an independent risk for low birth weight or preterm births. Mothers with more supportive social networks are found to experience better mental health outcomes [20]. While the association between neighborhood social support and maternal health outcomes has been examined by these studies, researchers have not examined the role of patientphysician communication as a possible intervening variable between social support and health outcome.

Our study explores the racial difference in prospective mothers' communication with healthcare providers, as quality communication between a physician and a patient positively influences the health outcomes and thus constitutes an integral part of health care [21]. To the best of our knowledge, our study is the first to explore social support's influence on health communication among Asian immigrant women in the US. The rising number of Asian immigrants in the US makes studies on Asian American women's maternal health even more important.

\section{Method}

We use the 2007 Los Angeles Mommy and Baby (LAMB) survey to study overall health disparities in maternal and 
child health before and during pregnancy. The 2007 LAMB survey was conducted to new mothers who had a live birth in the Los Angeles County four to seven months before. The survey contains items examining maternal and child health indicators such as prenatal care, health behaviour during pregnancy, and postnatal recovery. Participating mothers were recruited through multilevel sampling and were asked to fill out the sections of the survey that were relevant to their experience. For the purpose of this research, we analyzed survey items related to participating mothers' prepregnancy and prenatal visits to health providers and social environment. We use four sets of questionnaire items to construct four scales that measure different aspects of social environment, adapted from the Project on Human Development in Chicago Neighborhoods (PHDCN) [16] and the LAMB project team. In this study, we measure Asian women's and Caucasian women's neighborhood social environment by four related key variables: (1) instrumental and emotional support during pregnancy, (2) neighborhood social cohesion, (3) neighborhood social exchange, and (4) neighborhood services characterized by safety, friendliness, quietness, cleanliness, and public service.

2.1. Instrumental and Emotional Support during Pregnancy. The 2007 LAMB survey asked each respondent "During your last pregnancy, how often would you get these kinds of support, if you needed them?" and the respondent would choose from "Never," "Rarely," "Sometimes," "Most of the time," and "All of the time" for each of the following seven items:

(a) Someone to loan me $\$ 50$; (b) Someone to help me if I were sick and needed to be in bed; (c) Someone to take me to the clinic or doctor if I needed a ride; (d) Someone to give me a place to live; (e) Someone to help me with babysitting or child care; (f) Someone to help me with household chores; (g) Someone to talk to about my problems.

For each of the seven items, we coded this Likert scale as 1 through 5 with "Never" being 1 and "All of the time" being 5 and summed up the seven items to create a scale of prenatal social network support.

2.2. Neighborhood Social Cohesion. The 2007 LAMB survey asked each respondent "Do you agree that people in your neighborhood...

(a) Are willing to help their neighbors? (b) This is a close-knit (tight) neighborhood? (c) Can be trusted? (d) Generally do not get along with each other? (e) Do not share the same values?"

The respondent would choose from "Strongly disagree," "Disagree," "Neutral," "Agree," and "Strongly agree" for each of the above five items. For each of the first three items we coded this Likert scale as 1 through 5 with "Strongly disagree" being 1 and "Strongly agree" being 5, while coding the remaining two items in the reverse order as conceptually Item D and Item
E measure the antonymous construct of neighborhood social cohesion. We summed up the five items to create a scale for neighborhood social cohesion.

2.3. Neighborhood Social Exchange. The 2007 LAMB survey asked each respondent "And how often do your neighbors...

(a) Do favors for each other? (b) Ask each other advice about personal things such as child rearing or job openings? (c) Have parties or other get-togethers where other people in the neighborhood are invited? (d) Visit in each other's homes or on the street? (e) Watch over each other's property?"

and the respondent would choose from "Never," "Almost never," "Sometimes," "Fairly often," and "Very often" for each of the above five items. For each of the five items, we coded this Likert scale as 1 through 5 with "Never" being 1 and "Very often" being 5 and summed up the five items to create a scale of neighborhood social exchange.

2.4. Neighborhood Services. The 2007 LAMB survey asked each respondent "How would you rate this neighborhood in terms of its. .

(a) Police protection; (b) Protection of property; (c) Safety from violence; (d) Friendliness; (e) Cleanliness; (f) Quietness; (g) Quality of schools; (h) Availability of parks, playgrounds, or sidewalks; (i) Municipal services (e.g., trash pickup, road repair, libraries, water)"

and the respondent would choose from "Very poor," "Poor," "Sometimes," "Good," and "Very good" for each of the above nine items. For each of the nine items, we coded this Likert scale as 1 through 5 with "Very poor" being 1 and "Very good" being 5 and summed up the nine items to create a scale of neighborhood services.

2.5. Dependent Variables. We use the following three dummy variables to measure the communication between White/Asian women and health care providers: whether the respondent had talked to provider to prepare for pregnancy (women who did not do so were coded as 1 and women who did so were coded as 0 ); whether she talked about sensitive issues in preparatory visit prior to pregnancy (women who talked about domestic violence, anxiety/depression, or birth control during her prepregnancy visit were coded as 0 , and women who did not do so were coded as 1); whether she talked about stigmatized issues in prenatal visits (women who talked about domestic abuse, illicit drug, and HIV during her prenatal visits were coded as 0 while women who did not do so were coded as 1).

2.6. Logistic Regressions with Geographic Fixed Effects. We use six logistic regressions to examine the association between the social environment and women's communication with 
TABLE 1: Logistic regressions of neighborhood factors in predicting communication during prepregnancy and prenatal visit to health care providers.

\begin{tabular}{|c|c|c|c|c|c|c|}
\hline & \multicolumn{2}{|c|}{$\begin{array}{l}\text { Not talking to a health care } \\
\text { provider to get prepared for } \\
\text { pregnancy (prepregnancy } \\
\text { visits) }\end{array}$} & \multicolumn{2}{|c|}{$\begin{array}{c}\text { Not talking about } \\
\text { sensitive issues } \\
\text { (prepregnancy visits) }\end{array}$} & \multicolumn{2}{|c|}{$\begin{array}{l}\text { Not talking about } \\
\text { stigmatized issues } \\
\text { (prenatal visits) }\end{array}$} \\
\hline & Asian & Caucasian & Asian & Caucasian & Asian & Caucasian \\
\hline $\begin{array}{l}\text { Instrumental and emotional } \\
\text { support }\end{array}$ & \multicolumn{4}{|c|}{ No data was collected } & -0.023 & -0.017 \\
\hline Neighbor social cohesion & $0.065^{*}$ & -0.007 & 0.010 & 0.049 & 0.056 & 0.022 \\
\hline Neighbor social exchange & $-0.092^{* * *}$ & -0.027 & $-0.081^{* *}$ & $-0.045^{*}$ & -0.042 & -0.012 \\
\hline Neighbor services & -0.010 & -0.001 & 0.006 & 0.010 & $-0.036^{*}$ & -0.012 \\
\hline
\end{tabular}

Notes:

$(1){ }^{*}$ if $P<0.05,{ }^{* *}$ if $P<0.01,{ }^{* * *}$ if $P<0.001$

(2) controlled for the following covariates: age, education, income, and geographic units (operationalized as seven dummy variables accounting for the eight service planning areas in Los Angeles County).

health care providers (operationalized as the three abovementioned dependent variables), stratified by race (Caucasians versus Asians). To control for confounding factors, we use the respondent's age, education (dummy-coded by level of educational attainment), household income (dummycoded by 5 levels of household income), and geographic units (operationalized as seven dummy variables accounting for the eight service planning areas in Los Angeles County) as covariates in our logistic models.

\section{Results}

Table 1 summarizes the results of the six regressions. Neighborhood social exchange is negatively associated with not talking about sensitive issues in prepregnancy visits among Caucasian women (logged odds: $-0.045 ; P<0.01$ ) and Asian women (logged odds: $-0.081 ; P<0.001$ ), which indicates both Caucasian and Asian women living in a neighborhood with a higher level of social exchange tend to talk about sensitive issues in prepregnancy medical visits. However, the negative association between neighborhood social exchange and not talking to a health care provider to prepare for pregnancy is only significant among Asian women (logged odds: $-0.092 ; P<0.05$ ), meaning Asian women living in a neighborhood with higher level of social exchange tend to talk to their health care provider about preparing for pregnancy. Neighborhood social cohesion has a significant positive association with not talking to provider to prepare for pregnancy among Asians (logged odds: 0.065; $P<0.05$ ), which is a pattern that is not seen among Caucasian women (logged odds: $-0.007 ; P>0.05)$. During prenatal visits to health care providers, the only significant association exists between neighborhood services and not talking about stigmatized issues among Asian women (logged odds: -.036; $P<0.05)$, a pattern that is not statistically significant among Caucasian women (logged odds: $-.012 ; P>0.05$ ).

\section{Discussion}

As social support is significantly correlated with a woman's "maternal confidence" to provide infant care [22], it might be plausible to infer that such confidence could trigger the mother to skip physician visits before, during, and after her pregnancy. According to our study, Asian women with higher neighborhood social exchange are more likely to visit health care providers to prepare for pregnancy while Asian women from a more cohesive neighborhood sharing similar values are less likely to visit healthcare providers to prepare for pregnancy. This might indicate the feared "displacement effect" (word-of-mouth experience sharing in a more cohesive community displaces the resident's physician visits) could be happening in immigrant or minority neighborhoods, yet the reciprocal help among neighbors is unlikely to displace a woman's visit to health care providers in preparation for her pregnancy. This "displacement effect," however, is not evident among Caucasian women when it comes to their visit to health care providers. Future research could further examine ethnic differences in social support and maternal confidence in relation to women's possibility of making prepregnancy visits.

It is worth noting that Caucasian women and Asian women do share an important similarity in discussing sensitive issues such as domestic violence, anxiety, and depression during their prepregnancy visits to health care providers: those who enjoy more neighborhood social exchange are more likely to discuss these issues during their visit to healthcare providers to prepare for their pregnancy. While our study of associations cannot be used as causal evidence, this significant pattern shared by both Caucasian women and Asian women might give us a hypothesis for future experimental studies: living in an environment of friendliness and trust helps people open up about sensitive topics in their conversation with health care providers.

Caucasian and Asian women differ in their likelihood of discussing stigmatized issues (such as drinking, abuse, illegal drug use, and HIV/AIDS) during their prenatal visit. Better 
neighborhood services (characterized by safety, cleanness, and public services) are positively associated with Asian women's likelihood of discussing stigmatized issues with their healthcare providers during prenatal visits, while this association is much weaker and statistically insignificant among Caucasian women, albeit with the same sign of the logistic coefficient. Exactly why the pattern differs between Asians and Caucasians is unknown to us, but we do notice in our data that Caucasian women on average reported having significantly higher instrumental and emotional support level from their personal networks than Asian women. This might mean that Asian women with their lower level of instrumental and emotional support from their personal networks actually need neighborhood services more than their Caucasian counterparts to have a sense of trust and security for opening a conversation about stigmatized issues during pregnancy.

On average, Asian women in this study lived in neighborhoods with lower service quality than Caucasians. As our study shows that neighborhood service is a significant predictor of Asian women's discussion of stigmatized issues with health care providers (usually considered as a good sign in health communication), it could be critical to improve the safety, cleanness, and public service in neighborhoods with higher proportions of Asian population, as the improvement of the public service quality of those neighborhoods could be an indirect way to reduce the racial disparities in benefiting from maternal health services. From the perspective of planning interventions, initiatives like improving neighborhood services could be easier to implement than improving neighborhood social support or enhancing one's personal network, as the latter two tend to be individual choices and community culture rather than policy makers' actionable targets.

\section{Conflict of Interests}

The authors declare that there is no conflict of interests regarding the publication of this paper.

\section{References}

[1] M. van Ryn and S. S. Fu, "Paved with good intentions: do public health and human service providers contribute to racial/ethnic disparities in health?" American Journal of Public Health, vol. 93, no. 2, pp. 248-255, 2003.

[2] M. Kuppermann, E. Gates, and A. E. Washington, "Racialethnic differences in prenatal diagnostic test use and outcomes: preferences, socioeconomics, or patient knowledge?" Obstetrics \& Gynecology, vol. 87, no. 5 I, pp. 675-682, 1996.

[3] M. H. Davis, M. M. Morris, and L. A. Kraus, "Relationshipspecific and global perception of social support: associations with well-being and attachments," Journal of Personality and Social Psychology, vol. 74, no. 2, pp. 468-481, 1998.

[4] J. S. Ali, S. McDermott, and R. G. Gravel, "Recent research on immigrant health from statistics Canada's population surveys," Canadian Journal of Public Health, vol. 95, no. 3, pp. I9-I13, 2004.

[5] I. Hyman, "Negative consequences of acculturation on health behavior, social support and stress among pregnant Southeast
Asian immigrant women in Montreal: an exploratory study," Canadian Journal of Public Health, vol. 91, no. 5, pp. 357-360, 2000.

[6] W. P. Frisbie, Y. Cho, and R. A. Hummer, "Immigration and the health of Asian and pacific islander adults in the United States," American Journal of Epidemiology, vol. 153, no. 4, pp. 372-380, 2001.

[7] R. M. Perloff, B. Bonder, G. B. Ray, E. B. Ray, and L. A. Siminoff, "Doctor-patient communication, cultural competence, and minority health: theoretical and empirical perspectives," American Behavioral Scientist, vol. 49, no. 6, pp. 835-852, 2006.

[8] H. M. Bauer, M. A. Rodriguez, S. S. Quiroga, and Y. G. FloresOrtiz, "Barriers to health care for abused Latina and Asian immigrant women," Journal of Health Care for the Poor and Underserved, vol. 11, no. 1, pp. 33-44, 2000.

[9] M. R. Yoshioka and A. Schustack, "Disclosure of HIV status: cultural issues of asian patients," AIDS Patient Care and STDs, vol. 15, no. 2, pp. 77-82, 2001.

[10] D. Chin and K. W. Kroesen, "Disclosure of HIV infection among Asian/Pacific Islander American women: cultural stigma and support," Cultural Diversity \& Ethnic Minority Psychology, vol. 5, no. 3, pp. 222-235, 1999.

[11] G. Choi, "Acculturative stress, social support, and depression in Korean American families," Journal of Family Social Work, vol. 2, no. 1, pp. 81-97, 2013.

[12] S. Cohen and T. A. Wills, "Stress, social support, and the buffering hypothesis," Psychological Bulletin, vol. 98, no. 2, pp. 310-357, 1985.

[13] A. L. Coker, P. H. Smith, M. P. Thompson, R. E. McKeown, L. Bethea, and K. E. Davis, "Social support protects against the negative effects of partner violence on mental health," Journal of Women's Health \& Gender-based Medicine, vol. 11, no. 5, pp. 465-476, 2002.

[14] D. J. Bearison, N. Minian, and L. Granowetter, "Medical management of asthma and folk medicine in a hispanic community," Journal of Pediatric Psychology, vol. 27, no. 4, pp. 385-392, 2002.

[15] A. C. Kouzis and W. W. Eaton, "Absence of social networks, social support and health services utilization," Psychological Medicine, vol. 28, no. 6, pp. 1301-1310, 1998.

[16] A. L. Cradock, I. Kawachi, G. A. Colditz, S. L. Gortmaker, and S. L. Buka, "Neighborhood social cohesion and youth participation in physical activity in Chicago," Social Science \& Medicine, vol. 68, no. 3, pp. 427-435, 2009.

[17] S. L. Buka, R. T. Brennan, J. W. Rich-Edwards, S. W. Raudenbush, and F. Earls, "Neighborhood support and the birth weight of urban infants," American Journal of Epidemiology, vol. 157, no. 1, pp. 1-8, 2003.

[18] J. K. Rajaratnam, J. G. Burke, and P. O'Campo, "Maternal and child health and neighborhood context: the selection and construction of area-level variables," Health and Place, vol. 12, no. 4, pp. 547-556, 2006.

[19] S. Nkansah-Amankra, A. Dhawain, J. R. Hussey, and K. J. Luchok, "Maternal social support and neighborhood income inequality as predictors of low birth weight and preterm birth outcome disparities: analysis of South Carolina pregnancy risk assessment and monitoring system survey, 2000-2003," Maternal and Child Health Journal, vol. 14, no. 5, pp. 774-785, 2010.

[20] A. B. Balaji, A. H. Claussen, D. C. Smith, S. N. Visser, M. J. Morales, and R. Perou, "Social support networks and maternal mental health and well-being," Journal of Women's Health, vol. 16, no. 10, pp. 1386-1396, 2007. 
[21] L. M. L. Ong, J. C. J. M. De Haes, A. M. Hoos, and F. B. Lammes, "Doctor-patient communication: a review of the literature," Social Science and Medicine, vol. 40, no. 7, pp. 903-918, 1995.

[22] P. L. Ruchala and D. C. James, "Social support, knowledge of infant development, and maternal confidence among adolescent and adult mothers," Journal of Obstetric, Gynecologic, and Neonatal Nursing, vol. 26, no. 6, pp. 685-689, 1997. 


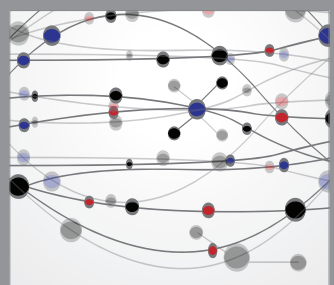

The Scientific World Journal
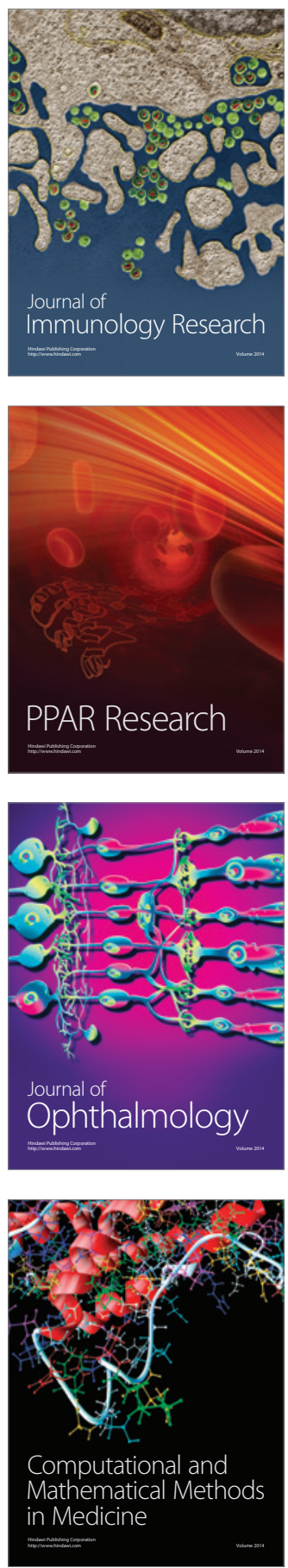

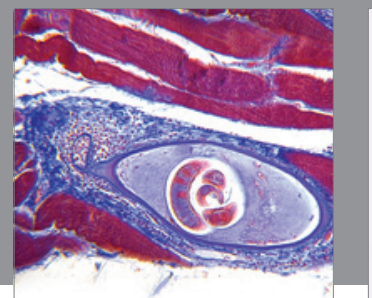

Gastroenterology

Research and Practice
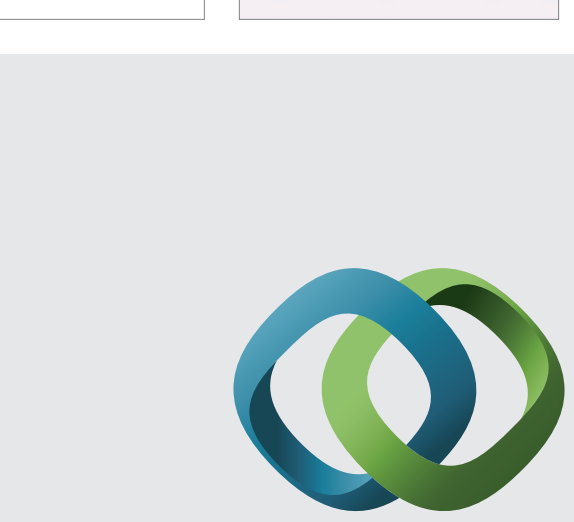

\section{Hindawi}

Submit your manuscripts at

http://www.hindawi.com
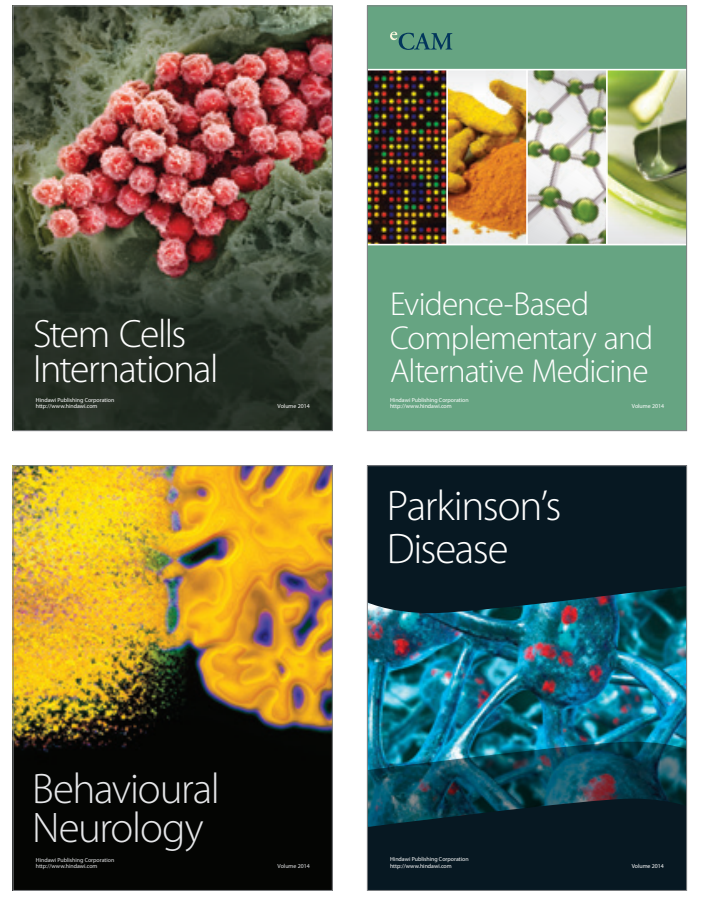
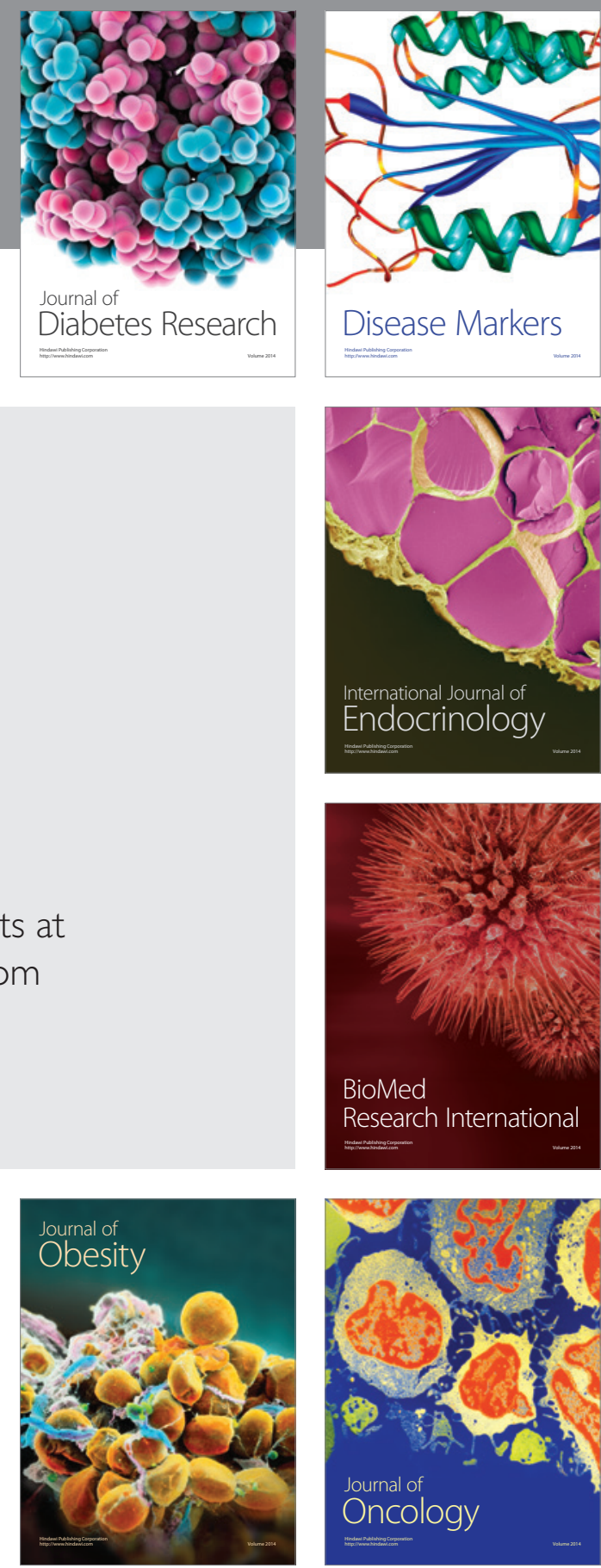

Disease Markers
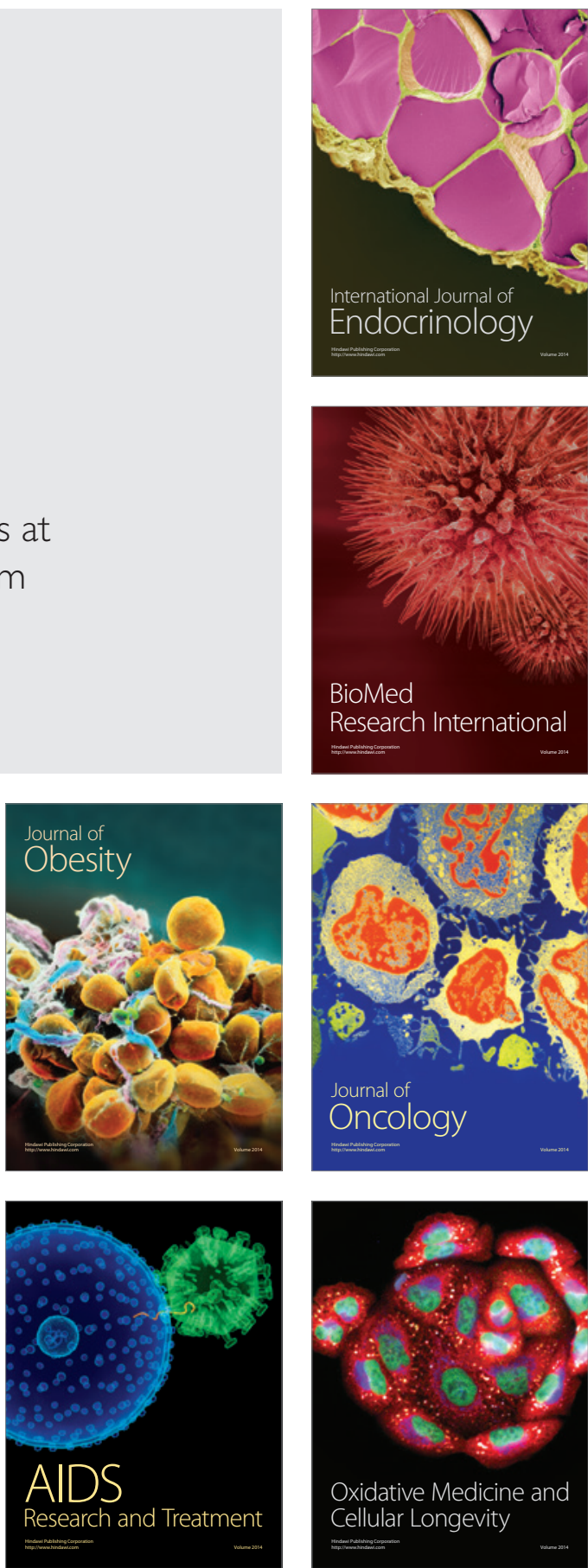